Bull. Austral. Math. Soc.

$46 \mathrm{~B} 03,46 \mathrm{~B} 20$

VOL. 56 (1997) [193-196]

\title{
ON A BANACH SPACE WITHOUT A WEAK MID-POINT LOCALLY UNIFORMLY ROTUND NORM
}

\author{
Zhibao Hu, Warren B. Moors and Mark A. Smith
}

\begin{abstract}
In this paper we show that (i) $l^{\infty}$ does not admit an equivalent weak mid-point locally uniformly rotund norm and (ii) $l^{\infty} / c_{0}$ does not admit an equivalent rotund norm.
\end{abstract}

\section{INTRODUCTION}

In [3], Lindenstrauss showed that $l^{\infty}$ does not admit an equivalent weakly locally uniformly rotund norm. In this paper, we refine his argument to show that $l^{\infty}$ does not even admit an equivalent weak mid-point locally uniformly rotund norm. In addition, our argument also shows that $l^{\infty} / c_{0}$ does not admit an equivalent rotund norm, a result previously proven by Bourgain in [2]. We say that a norm $\|\cdot\|$ on a Banach space $X$ is rotund if each point of the unit sphere $S(X)$ is an extreme point of the closed unit ball $B(X)$. Further, we say that a norm $\|\cdot\|$ is weak mid-point locally uniformly rotund (or weak MLUR for short) if for each $x \in X \backslash\{0\}$ and each sequence $\left\{h_{n}: n \in \mathbb{N}\right\}$ in $X, h_{n} \rightarrow 0$ weakly whenever $\lim _{n \rightarrow \infty}\left\|x \pm h_{n}\right\|=\|x\|$. In [4], it is shown that a norm $\|\cdot\|$ is weak MLUR if and only if each point of $S(X)$ (when considered as a subset of the second dual ball) is an extreme point of the second dual ball.

\section{THEOREM .}

(i) $l^{\infty}$ does not admit an equivalent weak MLUR norm;

(ii) $l^{\infty} / c_{0}$ does not admit an equivalent rotund norm.

ProOF: (i) Let $\|\cdot\|_{\infty}$ denote the usual sup norm on $l^{\infty}$ and let $\|\cdot \cdot\|$ denote any equivalent norm on $l^{\infty}$. By the support of $x \in l^{\infty}$ we mean the set $\sigma(x) \equiv\{k \in$ $\mathbb{N}: x(k) \neq 0\}$. Let $F_{0} \equiv\left\{x \in l^{\infty}:\|x\|_{\infty}=1\right.$ and $\mathbb{N} \backslash \sigma(x)$ is infinite $\}$. Let $m_{0} \equiv$ $\inf \left\{|\|x\||: x \in F_{0}\right\}$ and $M_{0} \equiv \sup \left\{\|x\|: x \in F_{0}\right\}$. As $\|\cdot\| \|$ is an equivalent norm on $l^{\infty}, 0<m_{0} \leqslant M_{0}<\infty$. We proceed by induction.

Received 1 October 1996

Copyright Clearance Centre, Inc. Serial-fee code: 0004-9729/97 \$A2.00+0.00. 
STEP 1. Choose $x_{1} \in F_{0}$ so that $\left(3 M_{0}+m_{0}\right) / 4 \leqslant\left\|x_{1}\right\|$ and choose distinct integers $i_{1}$ and $j_{1} \in \mathbb{N} \backslash \sigma\left(x_{1}\right)$. Then define $F_{1} \equiv\left\{x \in F_{0}: x\right.$ agree with $x_{1}$ on $\left.\sigma\left(x_{1}\right) \cup\left\{i_{1}, j_{1}\right\}\right\}$ and set $m_{1} \equiv \inf \left\{\|x\|: x \in F_{1}\right\}$ and $M_{1} \equiv \sup \left\{\|x\|: x \in F_{1}\right\}$.

Now, after the first $n$ steps of the induction, we shall have constructed elements $\left\{x_{1}, x_{2}, \ldots, x_{n}\right\} \subseteq F_{0} \subseteq l^{\infty}$, non-empty subsets $F_{n} \subseteq F_{n-1} \subseteq \ldots F_{1} \subseteq F_{0}$, positive real numbers $m_{0} \leqslant m_{1} \leqslant \ldots \leqslant m_{n-1} \leqslant m_{n} \leqslant M_{n} \leqslant M_{n-1} \leqslant \ldots \leqslant M_{1} \leqslant M_{0}$ and distinct positive integers $\left\{i_{1}, i_{2}, \ldots, i_{n}, j_{1}, j_{2}, \ldots, j_{n}\right\}$ such that, for each $k$, $(1 \leqslant k \leqslant n)$;

(a) $x_{k} \in F_{k-1},\left(3 M_{k-1}+m_{k-1}\right) / 4 \leqslant\left\|x_{k}\right\|$;

(b) $\sigma\left(x_{k}\right) \cap\left\{i_{1}, i_{2}, \ldots, i_{k}, j_{1}, j_{2}, \ldots, j_{k}\right\}=\emptyset$;

(c) $F_{k} \equiv\left\{x \in F_{0}: x\right.$ agrees with $x_{k}$ on $\left.\sigma\left(x_{k}\right) \cup\left\{i_{1}, i_{2}, \ldots, i_{k}, j_{1}, j_{2}, \ldots, j_{k}\right\}\right\}$;

(d) $m_{k} \equiv \inf \left\{\|x\|: x \in F_{k}\right\}$ and $M_{k} \equiv \sup \left\{\|x\|: x \in F_{k}\right\}$.

STEP $n+1$. Choose $x_{n+1} \in F_{n}$ so that $\left(3 M_{n}+m_{n}\right) / 4 \leqslant\left\|x_{n+1}\right\|$ and choose distinct integers $i_{n+1}$ and $j_{n+1} \in \mathbb{N} \backslash\left(\sigma\left(x_{n+1}\right) \cup\left\{i_{1}, i_{2}, \ldots, i_{n}, j_{1}, j_{2}, \ldots, j_{n}\right\}\right)$. Then define $F_{n+1} \equiv\left\{x \in F_{0}: x\right.$ agrees with $x_{n+1}$ on $\left.\sigma\left(x_{n+1}\right) \cup\left\{i_{1}, i_{2}, \ldots, i_{n+1}, j_{1}, j_{2}, \ldots, j_{n+1}\right\}\right\}$ and set $m_{n+1} \equiv \inf \left\{\|x\| \|: x \in F_{n+1}\right\}$ and $M_{n+1} \equiv \sup \left\{\|x\|: x \in F_{n+1}\right\}$. This completes the induction.

For each $n \in \mathbb{N}$, define $h_{n} \in l^{\infty}$ by

$$
h_{n}(k) \equiv \begin{cases}1 & \text { if } k \in\left\{i_{n}, i_{n+1}, \ldots\right\} \\ 0 & \text { otherwise }\end{cases}
$$

Also define $x_{\infty} \in l^{\infty}$ by

$$
x_{\infty}(k) \equiv \begin{cases}x_{n}(k) & \text { if } k \in \sigma\left(x_{n}\right) \text { for some } n \\ 0 & \text { otherwise. }\end{cases}
$$

It is readily verfied that $x_{\infty}$ is well-defined and that $x_{\infty} \in \bigcap\left\{F_{n}: n \in \mathbb{N}\right\}$. It is also clear that $x_{\infty} \pm h_{n+1} \in F_{n}$ for each $n \in \mathbb{N}$. Next, choose $f \in\left(l^{\infty}\right)^{*}$ so that $f\left(h_{1}\right)=\|f\|_{\infty}=1$ and $f(y)=0$ for each $y \in c_{0}$. Clearly, for such an element $f$, we have that $f\left(h_{n}\right)=1$ for all $n \in \mathbb{N}$. We complete the proof of part (i) by showing that $\lim _{n \rightarrow \infty}\left\|x_{\infty} \pm h_{n}\right\|=\|\| x_{\infty}\|\|$. To see this, observe that $2 x_{n}-F_{n} \subseteq F_{n}$ for each $n$. This, of course, implies that $\left\|2 x_{n}-y\right\| \leqslant M_{n}$ for each $y \in F_{n}$, and this in turn implies that $3 M_{n-1} / 2+m_{n-1} / 2 \leqslant\left\|2 x_{n}\right\| \leqslant M_{n}+\|y\|$ for each $y \in F_{n}$. Now, by taking the infimum over $y \in F_{n}$, we get that $3 M_{n-1} / 2+m_{n-1} / 2 \leqslant M_{n}+m_{n} \leqslant M_{n-1}+m_{n}$ and so $\left(M_{n-1}+m_{n-1}\right) / 2 \leqslant m_{n} \leqslant M_{n} \leqslant M_{n-1}$.

Therefore, $0 \leqslant\left|\left\|x_{\infty} \pm h_{n+1}\right\|\|-\| x_{\infty}\|\|\right| \leqslant M_{n}-m_{n} \leqslant\left(M_{n-1}-m_{n-1}\right) / 2$, since $x_{\infty} \pm h_{n+1}$ and $x_{\infty} \in F_{n}$. Hence, by induction, $0 \leqslant\left|\left\|\left|x_{\infty} \pm h_{n+1}\left\|\left|-\left\|x_{\infty}\right\|\right| \mid \leqslant\right.\right.\right.\right.$ $\left(M_{0}-m_{0}\right) / 2^{n} ;$ which shows that $\lim _{n \rightarrow \infty}\left\|x_{\infty} \pm h_{n}\right\|=\left\|x_{\infty}\right\| \|$. 
(ii) Let $\left\||\||\right.$ be any equivalent norm on $l^{\infty} / c_{0}$ and let $\pi: l^{\infty} \rightarrow l^{\infty} / c_{0}$ denote the usual quotient mapping. We apply the construction from part (i) to the equivalent norm (on $l^{\infty}$ ) $\|x\| \equiv\|x\|_{\infty}+\|\pi(x)\| \|$. Indeed, from part (i) we have the existence of an element $x_{\infty} \in l^{\infty}$ and a sequence $\left\{h_{n}: n \in \mathbb{N}\right\} \subseteq l^{\infty}$ such that $\lim _{n \rightarrow \infty}\left\|x_{\infty} \pm h_{n}\right\|=$ $\left\|x_{\infty}\right\|,\left\|x_{\infty} \pm h_{n}\right\|_{\infty}=\left\|x_{\infty}\right\|_{\infty}=1$ for all $n$ and $\pi\left(h_{n}\right)=\pi\left(h_{1}\right) \neq 0$ for each $n$. Therefore, $\left\|\pi\left(x_{\infty}\right)\right\|\|=\| \pi\left(x_{\infty}\right) \pm \pi\left(h_{1}\right) \|$; which shows that the $\|\cdot\| \cdot \|$ norm on $l^{\infty} / c_{0}$ is not rotund.

COROLLARY . $l^{\infty}$ cannot be equivalently renormed so that its unit sphere (considered as a subset of the second dual ball) is an extremal subset of its second dual ball.

ProOF: Suppose to the contrary that such a norm exists. Call it $\|\cdot\|_{1}$ say. Let $\|\cdot\|_{2}$ be any equivalent rotund norm on $l^{\infty}$ and define $\|\cdot\| \cdot \|: l^{\infty} \rightarrow R$ by $\|x\|\|\equiv\| x\left\|_{1}+\right\| x \|_{2}$. It is easy to check that each point of the unit sphere of the $\|\cdot\| \|$ norm is an extreme point of its second dual ball and so the $\|\cdot|\||$ norm is weak MLUR. But this contradicts the above Theorem. Therefore, no such norm exists.

REMARK. If $\|\cdot\| \cdot \|$ is an equivalent Kadec norm then its unit sphere is an extremal subset of its second dual ball. Hence, $l^{\infty}$ does not admit an equivalent Kadec norm.

Note ADDED IN PROOF: It has recently come to the attention of the authors that the paper $\left[\mathbf{1}\right.$ ] contains a proof of the fact that $l^{\infty}$ does not admit an equivalent weak mid-point locally uniformly rotund norm.

\section{REFERENCES}

[1] G.A. Aleksandrov and I.P. Dimitrov, 'On the equivalent weakly midpoint locally uniformly rotund renorming of the space $l^{\infty}$, (in Russian), in Mathematics and Mathematical Education (Sunny Beach, 1985), pp. 189-191.

[2] J. Bourgain, ' $l \infty / c_{0}$ has no equivalent strictly convex norm', Proc. Amer. Math. Soc. 78 (1980), 225-226.

[3] J. Lindenstrauss, 'Weakly compact sets - their topological properties and the Banach spaces they generate', Ann. of Math. Studies 69 (1972), 235-273.

[4] M.A. Smith, 'A curious generalisation of local uniform rotundity', Comment Math. Univ. Carol 25 (1984), 659-665.

Division of Mathematics

Science and Exss

El Paso Community College

Valle Verde Campus

PO Box 20500 El Paso

Texas 79998

United States of America
Department of Mathematics The University of Auckland Private Bag 92019 Auckland New Zealand 
Department of Mathematics and Statistics Miami University

Oxford

Ohio 45056

United States of America 